\title{
Pitfalls in the Diagnosis and Treatment of Non-Traumatic Hemoperitoneum
}

Tatsuma Fukuda ${ }^{1,2 *}$,Toshiaki Mochizuki ${ }^{2}$,Norio Otani ${ }^{2}$,Naoki Yahagi' and Shinichi Ishimatsu ${ }^{2}$

${ }^{1}$ Department of Emergency and Critical Care Medicine, Graduate School of Medicine, The University of Tokyo, Japan

${ }^{2}$ Department of Emergency and Critical Care Medicine, St.Luke's International Hospital, Japan

\begin{abstract}
Non-traumatic hemoperitoneum is often caused by vascular lesions. In some of the diseases which cause vascular lesions, the lesions are presented systemically. We report a 34-year-old man with non-traumatic hemoperitoneum due to dissection of splenic artery. He had systemic vascular lesions, including a dissecting aneurysm in left common iliac artery. We found the iliac arterial lesion in advance; therefore we could avoid potentially lethal complications accompanied by examination and treatment of the splenic artery lesion. In non-traumatic hemoperitoneum, it is important to confirm that other vascular lesions are not present between the puncture site for cannulation and target lesion.
\end{abstract}

Keywords: Non-traumatic hemoperitoneum; Vascular EhlersDanlos syndrome; Vasculitis mimics; Angiography; Transcatheter Arterial Embolization (TAE)

\section{Introduction}

With regard to the causes of non-traumatic hemoperitoneum, one must consider diseases that cause vascular lesions $[1,2]$ some of which are systemic or multifocal [1-3]. Here, we describe a non-traumatic hemoperitoneum case in which the presence of dissection of splenic artery led to the finding of systemic vascular lesions. In the diagnosis and treatment of non-traumatic hemoperitoneum, this instructive case reminded us of the need to fully consider the possibility of systemic vascular lesions during examination and treatment, or angiography and Transcatheter Arterial Embolization (TAE). It is important to confirm that other vascular lesions are not present between the puncture site for cannulation and target lesion. This case report is the first to present medical practice guidelines for avoiding the pitfalls of diagnosing and treating non-traumatic hemoperitoneum.

\section{Case Report}

A 34-year-old man was transported to our emergency medical center after experiencing left abdominal pain and fainting. The abdominal pain appeared while he was at work without known provocation, followed by intense pain and nausea; he then quickly fainted. He had a history of blood transfusion, with anemia (haemoglobin: $4.8 \mathrm{~g} / \mathrm{dL}$ ) of unknown etiology at the age of 20 and surgery for bloody stool of unknown etiology suspected in Meckel's diverticulum at the age of 30 years. The family history did not include any particular indications of any genetic disorders.

When the emergency services team made contact, the patient was disoriented and in a state of shock with a blood pressure of 74/46 mmHg. Upon his arrival at the emergency medical center, he was substantially lucid with a blood pressure of $96 / 60 \mathrm{mmHg}$, pulse of 81 beats $/ \mathrm{min}$, body temperature of $35.6^{\circ} \mathrm{C}$, respiration of 18 breaths $/ \mathrm{min}$, and $\mathrm{SpO}_{2}$ of $100 \%$ (oxygen $6 \mathrm{~L} / \mathrm{min}$ ); he responded favourably to transfusion. On physical examination, both pupils had a diameter of $5 \mathrm{~mm}$ and displayed a prompt light reflex. Signs of anemia were observed in the palpebral conjunctiva. Tenderness was observed in the left abdomen, but there were no signs of peritoneal irritation. The body surface lacked fresh bruise marks or subcutaneous bleeding suggestive of trauma.

Blood tests showed no obvious abnormal findings. Abdominal ultrasonography revealed a large amount of fluid accumulation in the abdominal cavity. Abdominal contrast Computed Tomography (CT)
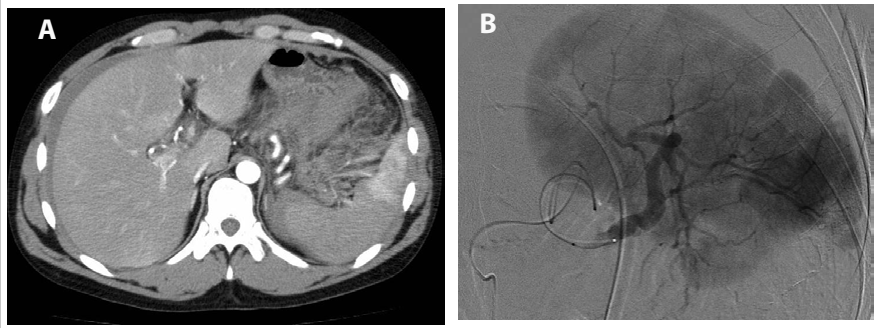

Figure 1: Imaging studies on admission.

a. Abdominal contrast CT showed a poor contrast effect of the part of the spleen. There were no findings of extravasation.

b. Angiography showed no findings of extravasation. A contrast deficit was observed in the lower pole of the spleen and wedge-shaped luminal stenosis was seen in the distal splenic artery.

imaging results showed that a large amount of fluid was accumulated in the abdominal cavity and an irregularly shaped high-absorption region was observed in the center of the retroperitoneal space extending from the peripancreatic area to the splenic hilum and the posterior wall of the stomach. Although no findings indicated extravasation of the contrast agent out of the blood vessels, part of the spleen showed a poor contrast effect (Figures 1a), so we suspected damage to the splenic artery and associated hemorrhaging. Abdominal contrast CT results led to the incidental finding of a dissecting aneurysm in the left common iliac artery (Figure 2a).

An emergency angiography was performed to diagnose and treat the splenic artery lesion with the right femoral artery cannulated to avoid an approach from the left femoral artery. Although the angiographic procedure showed no findings of extravasation of the contrast agent

*Corresponding author: Tatsuma Fukuda, Department of Emergency and Critical Care Medicine, Graduate School of Medicine, The University of Tokyo, 7-3-1, Hongo, Bunkyo-ku, Tokyo, 113-8655, Japan, Tel: +81 33815 5411; Fax: +813 3814 6446; E-mail: tatsumafukuda-jpn@umin.ac.jp

Received November 19, 2012; Accepted December 11, 2012; Published December 13, 2012

Citation: Fukuda T, Mochizuki T, Otani N,Yahagi N, Ishimatsu S (2013) Pitfalls in the Diagnosis and Treatment of Non-Traumatic Hemoperitoneum. J Vasc Med Surg 1: 102. doi:10.4172/2329-6925.1000102

Copyright: ( 2013 Fukuda T, et al. This is an open-access article distributed under the terms of the Creative Commons Attribution License, which permits unrestricted use, distribution, and reproduction in any medium, provided the original author and source are credited. 

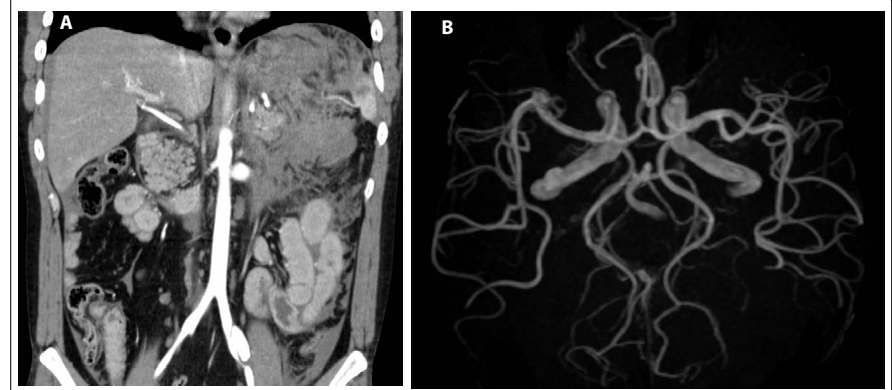

Figure 2: Systemic vascular lesions.

a. Contrast CT showed a dissecting aneurysm in the left common iliac artery. b. Magnetic Resonance angiography showed an unruptured cerebral aneurysm.
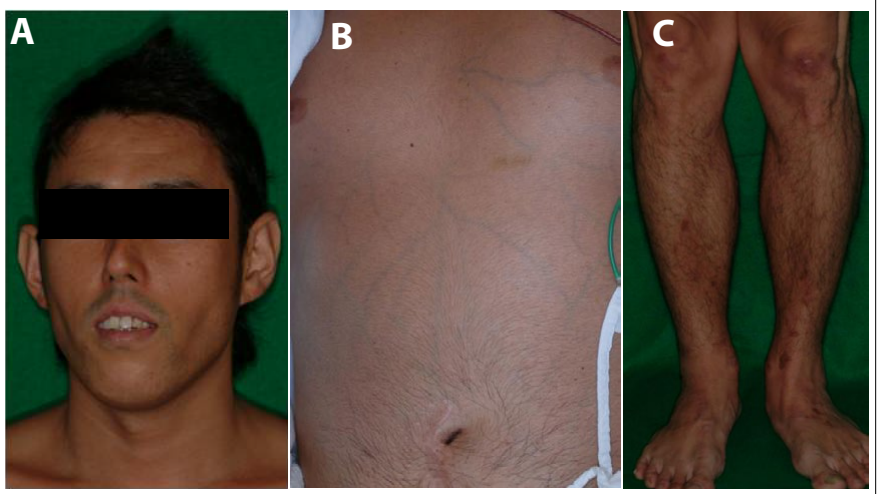

Figure 3: Characteristic physical appearance

a. He presented characteristic bird-like face, such as thin lips, thin philtrum, small chin, thin nose, and large eyes.

b. His veins under the skin were distinctly visible as the skin was translucent on the thorax.

c. Atrophic scarrings and purpura were seen in his legs.

from the blood vessels, a contrast deficit was observed in the lower pole of the spleen and a wedge-shaped luminal stenosis was seen in the distal splenic artery (Figure 1b). Apparent aneurysm or dissection was absent, but this site was thought to have hemorrhaging caused by arterial dissection as well as obstruction of the lower pole branches by pseudo-cavity thrombosis.

With no extravasation findings and with stabilization of the hemodynamics after the patient's hospital arrival, there was no need to conduct Transcatheter Arterial Embolization (TAE), so a conservative approach was adopted for observing the patient's progress.

In the present case, dissecting aneurysm in the left common iliac artery was observed in addition to the splenic arterial lesion, and the patient had a history of anemia and bloody stools, both with unknown etiology. Therefore, we suspected the presence of a disease that would cause abnormalities in the blood vessels. When a full-body inspection of the blood vessels was conducted, other vascular lesions were found, such as unruptured cerebral aneurysm (Figure 2b), narrowed and tortuous sites of the hepatic artery, and irregularities in the internal carotid arterial wall. The presence of vasculitis or vasculitis-like noninflammatory disorder was suspected [3]. Vascular Ehlers-Danlos syndrome was strongly suspected given the clinical features including multiple vascular lesions, a history of gastrointestinal bleeding, the characteristic facial appearance (thin lips and philtrum, small chin, thin nose, large eyes), and skin findings (translucent visible of the subcutaneous veins, atrophic scarring, purpura [4-11] (Figures 3a-3c) Identification of COL3A1 genetic mutation is necessary for a definitive diagnosis, but a genetic examination was not permitted after discussion between the patient and genetic counsellors resulted, thus genetic evidence could not be obtained [4-7,9,10].

Contrast CT performed on day 3 to enable full-body scrutiny of the blood vessels yielded a uniformly favourable contrast effect in the spleen. Believing that blood flow in the lower pole branches of the splenic artery had recanalized, we performed TAE on day 4 , considering the risk of recurrent hemorrhaging. Irregular dilation at the wedge-shaped luminal stenosis of the distal splenic artery observed in the previous examination results was also observed by angiography, and resumption of blood flow was found at the lower pole branches. Arterial dissection was still believed to have occurred. TAE using a coil was performed on the proximal splenic artery trunk from the lower pole branch bifurcation. Blood flow to the spleen from the collateral circulation through the short gastric arteries persisted.

The patient's progress was favourable after TAE, and he was discharged on day 12 . The dissecting aneurysm in the left common iliac artery and unruptured intracranial aneurysm were treated using vascular surgery and neurosurgery, respectively.

\section{Discussion}

In the present case, splenic artery lesion hemorrhage was thought to be the cause of non-traumatic hemoperitoneum on the basis of images taken at the initial visit. As was the case with this patient, even when the culprit lesion is inferred to be a splenic artery lesion, it is not uncommon for the cause of the splenic artery lesion to be unknown at the initial visit. As the need to engage in treatment even while the etiology remains unknown, it is necessary to consider the possibility of systemic vascular lesions such as aneurysms.

In the present case, this was not our indication for examination, but a dissecting aneurysm in the left common iliac artery was discovered incidentally through abdominal contrast CT. All punctures in examination and treatment could thus be performed from the right femoral artery, allowing us to avoid potentially lethal complications.

Although not confirmed by genetic diagnosis in this case, the clinical features were highly suggestive of vascular Ehlers-Danlos syndrome. Vascular Ehlers-Danlos syndrome lesions can occur systemically in any of the blood vessels $[4,5,7]$. Vascular lesions can also present in the form of an aneurysms, dissections, or ruptures [4,5], but they can also occur without any of these [7]. Further, approximately $25 \%$ of patients with vascular Ehlers-Danlos syndrome are reported to have vascular lesions in the limbs (iliac artery, femoral artery, etc. [4]. Furthermore, although not limited to vascular Ehlers-Danlos syndrome, intraperitoneal aneurysms have been reported to occur in the aorta, iliac artery, and splenic artery, in order of frequency [12].

Upon encountering non-traumatic hemoperitoneum when the underlying disease is unknown, as in the present case, there is no choice but to treat the patient without a clear diagnosis. When angiography or TAE are conducted on the culprit lesion in the hemoperitoneum under such conditions, one must not forget the possibility of vascular lesions being present at other sites.

It is important to confirm that other vascular lesions are not present between the arterial puncture site for cannulation and the targeted lesion. Specifically, it may be useful to refer to contrast CT scans that 
Citation: Fukuda T, Mochizuki T, Otani N,Yahagi N, Ishimatsu S (2013) Pitfalls in the Diagnosis and Treatment of Non-Traumatic Hemoperitoneum. J Vasc Med Surg 1: 102. doi:10.4172/2329-6925.1000102

Page 3 of 3

include all areas between the lesion site and the arterial puncture site [13]. In the event that CT imaging is not available, ultrasonography may also be useful [13-15].

Our report has some limitations. Currently, the percentage of non-traumatic hemoperitoneum cases caused by vascular lesions, percentage of illnesses causing vascular lesions with multiple lesions, and, in particular, percentage of cases in which at least one vascular lesion is present and involve an aortic or iliac arterial lesion are not known. With non-traumatic hemoperitoneum, it is very important to adopt an attitude of always considering the possible presence of multiple vascular lesions to avoid potentially lethal complications from examination or treatment. However, whether this is something that always must be determined is a question that must be considered according to statistical epidemiological data. To that end, we hope to conduct a large-scale epidemiological study on systemic vascular lesions in future.

\section{References}

1. Furlan A, Fakhran S, Federle MP (2009) Spontaneous abdominal hemorrhage: causes, CT findings, and clinical implications. AJR Am J Roentgenol 193: 1077 1087.

2. Lucey BC, Varghese JC, Soto JA (2005) Spontaneous hemoperitoneum: causes and significance. Curr Probl Diagn Radiol 34: 182-195.

3. Molloy ES, Langford CA (2008) Vasculitis mimics. Curr Opin Rheumatol 20: 29-34.

4. Pepin M, Schwarze U, Superti-Furga A, Byers PH (2000) Clinical and genetic features of Ehlers-Danlos syndrome type 4, the vascular type. N Engl J Med 342: 673-680.

5. Germain DP (2007) Ehlers-Danlos syndrome type 4. Orphanet Journal of Rare Diseases 2: 32.

6. Beighton P, De Paepe A, Steinmann B, Tsipouras P, Wenstrup RJ (1998) Ehlers-Danlos syndrome: revised nosology, Villefranche, 1997. Ehlers-Danlos National Foundation (USA) and Ehlers-Danlos Support Group (UK). Am J Med Genet 77: 31-37.

7. Beridze N, Frishman WH (2012) Vascular Ehlers-Danlos syndrome: pathophysiology, diagnosis, and prevention and treatment of its complication. Cardiol Rev 20: 4-7.

8. Germain DP (2006) The vascular Ehlers-Danlos syndrome. Curr Treat Options Cardiovasc Med 8: 121-127.

9. Germain DP, Herrera-Guzman Y (2004) Vascular Ehlers-Danlos syndrome Ann Genet 47: 1-9.

10. Germain DP (2002) Clinical and genetic features of vascular Ehlers-Danlos syndrome. Ann Vasc Surg 16: 391-397.

11. Cikrit DF, Miles JH, Silver D (1987) Spontaneous arterial perforation: the Ehlers-Danlos specter. J Vasc Surg 5: 248-255.

12. Spittel JA, Fairbairn JF, Kincaid OW, ReMine WH (1961) Aneurysm of the splenic artery. JAMA 175: 452-456.

13. Mortele KJ, Cantisani V, Brown DL, Ros PR (2003) Spontaneous intraperitoneal hemorrhage: imaging features. Radiol Clin North Am 41: 1183-1201.

14. Marcus R, Edell SL (1980) Sonographic evaluation of iliac artery aneurysms. Am J Surg 140: 666-670.

15. Gooding GA (1980) Ultrasonography of the iliac arteries. Radiology 135: 161 